\title{
Dynamic Economic Systems with Two Time Delays
}

\author{
Akio Matsumoto ${ }^{1}$, Ferenc Szidarovszky ${ }^{2}$ \\ ${ }^{1}$ Department of Economics, Chuo University, Higashi-Nakano, Hachioji, Tokyo, Japan \\ ${ }^{2}$ Department of Applied Mathematics, University of Pécs, Ifjúság, u. 6, Pécs, Hungary \\ Emal address: \\ akiom@tamacc.chuo-u.ac.jp (A. Matsumoto), szidarka@gmail.com (F. Szidarovszky)
}

\section{To cite this article:}

Akio Matsumoto, Ferenc Szidarovszky. Dynamic Economic Systems with Two Time Delays. International Journal of Economic Behavior and Organization. Special Issue: Recent Developments of Economic Theory and Its Applications. Vol. 3, No. 2-1, 2015, pp. 77-85.

doi: 10.11648/j.ijebo.s.2015030201.22

\begin{abstract}
An elementary analysis is developed to determine the stability region of certain classes of ordinary differential equations with two delays. Our analysis is based on determining stability switches first where an eigenvalue is pure complex, and then checking the conditions for stability loss or stability gain. In the cases of both stability losses and stability gains Hopf bifurcation occurs giving the possibility of the birth of limit cycles.
\end{abstract}

Keywords: Multiple Delays, Monopoly Model, Multiplier-Accelerator Model, Double-Edged Effect on Stability

\section{Introduction}

Delay differential equations have many applications in quantitative sciences including economics, biology, engineering among others. The single delay case is well established in the literature (Hayes, 1950, Bellman and Cooke 1963, Matsumoto and Szidarovszky, 2013a), however the presence of a second delay makes the models much more complicated. The works of Hale (1979) and Hale and Huang (1993) can be considered as major contributions. Matsumoto and Szidarovszky (2012) developed a simple analytic method, which is limited to examine only some special model variants. $\mathrm{Gu}$ et al. (2005) developed a geometric approach applicable for analyzing a more general class of models.

In this paper two particular models are examined and the two major approaches illustrated. A brief simulation study illustrates the theoretical findings.

\section{Model 1}

We first extend a text-book model of monopoly in microeconomics, following Matsumoto and Szidarovszky (2013b). A single product monopoly sells its product to a homogeneous market. Let $q$ denote the output of the firm, $p(q)=a-b q$ the price function and $C(q)=c q$ the cost function. Since $p(0)=0$ and $|\partial p(q) / \partial q|=b$, we call $a$ the maximum price and $b$ the marginal price. It is assumed that the firm knows the marginal price but does not know the maximum price. In consequence it has only an estimate of it at each time period, which is denoted by $a^{e}(t)$. So the firm believes that its profit is

$$
\left.\pi^{e}(t)=\left[a^{e}(t)-b q(t)\right] q(t)-c q(t)\right)
$$

and its best response is

$$
q^{e}(t)=\frac{a^{e}(t)-c}{2 b}
$$

Further, the firm expects the market price to be

$$
p^{e}(t)=a^{e}(t)-b q^{e}(t)=\frac{a^{e}(t)+c}{2} .
$$

However, the actual market price is determined by the real price function

$$
p^{a}(t)=a-b q^{e}(t)=\frac{2 a-a^{e}(t)+c}{2} .
$$

Using these price data, the firm updates its estimate. If the firm revises the estimate in such a way that the growth rate of the estimate is proportional to the difference between the expected and actual prices, the adjustment or learning process can be modeled by the differential equation

$$
\frac{d a^{e}(t) / d t}{a^{e}(t)}=k\left[p^{a}(t)-p^{e}(t)\right]
$$

where $k>0$ is the speed of adjustment. Substituting (3) and (4) reduces this to a differential equation with respect to $a^{e}$ as

$$
\frac{d a^{e}(t)}{a^{e}(t)}=k\left[a-a^{e}(t)\right]
$$


or multiplying both sides by $a^{e}(t)$ generates the logistic model

$$
\frac{d a^{e}(t)}{d t}=k a^{e}(t)\left[a-a^{e}(t)\right]
$$

which is a nonlinear differential equation.

If the firm uses two past price information, then the differential equation turns to be the delay differential equation

$$
\frac{d a^{e}(t)}{d t}=k a^{e}(t)\left[a-A^{\prime} a^{e}(t-\delta)-B^{\prime} a^{e}(t-\eta)\right]
$$

where $A^{\prime}>0$ and $B^{\prime}>0$ are positive constants while $\delta$ and $\eta$ denote the delays in the price information. It is clear that unique stationary state of this equation is $a$. By introducing the new variable, $x(t)=a^{e}(t)-a$, equation (7) is written as

$$
\dot{x}(t)+A x(t-\delta)+B x(t-\eta)=0
$$

where $A=a k A^{\prime}$ and $B=a k B^{\prime}$ are positive constants. We will first examine the asymptotical stability of the delay differential equation

The characteristic equation can be obtained by looking for the solution in the exponential form $u e^{\lambda t}$. By substitution,

$$
u \lambda e^{\lambda t}+A u e^{\lambda(t-\delta)}+B u e^{\lambda(t-\eta)}=0
$$

or

$$
\lambda+A e^{-\lambda \delta}+B e^{-\lambda \eta}=0
$$

Introduce the new variables

$$
\begin{gathered}
\omega=\frac{A}{A+B}, 1-\omega=\frac{B}{A+B}, \bar{\lambda}=\frac{\lambda}{A+B} \\
\gamma_{1}=\delta(A+B) \text { and } \gamma_{2}=\eta(A+B)
\end{gathered}
$$

to reduce equation (9) to the following:

$$
\bar{\lambda}+\omega e^{-\bar{\lambda} \gamma_{1}}+(1-\omega) e^{-\bar{\lambda} \gamma_{2}}=0 .
$$

Because of symmetry we can assume that $\omega \geq 1 / 2$. In order to find the stability region in the $\left(\gamma_{1}, \gamma_{2}\right)$ plane we will first characterize the cases when an eigenvalue is pure complex, that is, when $\bar{\lambda}=i v$. We can assume that $v>0$, since if $\bar{\lambda}$ is an eigenvalue, its complex conjugate is also an eigenvalue. Substituting $\bar{\lambda}=i v$ into equation (10) we have

$$
i v+\omega e^{-i v \gamma_{1}}+(1-\omega) e^{-i v \gamma_{2}}=0
$$

If there is no delay, then $\gamma_{1}=\gamma_{2}=0$ and equation (10) becomes

$$
\bar{\lambda}+1=0
$$

with a negative eigenvalue $\bar{\lambda}=-1$, so the system is asymptotically stable.

In the special case of $\gamma_{1}=0$, the equation becomes

$$
i v+\omega+(1-\omega) e^{-i v \gamma_{2}}=0
$$

The real and imaginary parts imply that

$$
\begin{aligned}
& \omega+(1-\omega) \cos \left(v \gamma_{2}\right)=0 \\
& v-(1-\omega) \sin \left(v \gamma_{2}\right)=0
\end{aligned}
$$

We can assume first $\omega>1 / 2$, so from the first equation

$$
\cos \left(v \gamma_{2}\right)=-\frac{\omega}{1-\omega}<-1
$$

so no stability switch is possible. If $\omega=1 / 2$, then

$$
\cos \left(v \gamma_{2}\right)=-1
$$

implying that $\sin \left(v \gamma_{2}\right)=0$ and so $v=0$ showing that there is no pure complex root. Hence for $\gamma_{1}=0$ the system is asymptotically stable with all $\gamma_{2} \geq 0$.

Assume now that $\gamma_{1}>0, \quad \gamma_{2} \geq 0$. The real and imaginary parts give two equations:

$$
\omega \cos \left(v \gamma_{1}\right)+(1-\omega) \cos \left(v \gamma_{2}\right)=0
$$

and

$$
v-\omega \sin \left(v \gamma_{1}\right)-(1-\omega) \sin \left(v \gamma_{2}\right)=0 .
$$

We consider the case of $\omega>1 / 2$ first and the symmetric case of $\omega=1 / 2$ will be discussed later. Introduce the variables

$$
x=\sin \left(v \gamma_{1}\right) \text { and } y=\sin \left(v \gamma_{2}\right)
$$

then (11) implies that

$$
\omega^{2}\left(1-x^{2}\right)=(1-\omega)^{2}\left(1-y^{2}\right)
$$

or

$$
-\omega^{2} x^{2}+(1-\omega)^{2} y^{2}=1-2 \omega .
$$

From (12),

$$
v-\omega x-(1-\omega) y=0
$$

implying that

$$
y=\frac{v-\omega x}{1-\omega} .
$$

Combining (13) and (14) yields

$$
-\omega^{2} x^{2}+(1-\omega)^{2}\left(\frac{v-\omega x}{1-\omega}\right)^{2}=1-2 \omega
$$

from which we can conclude that 


$$
x=\frac{v^{2}+2 \omega-1}{2 v \omega}
$$

and then from (14),

$$
y=\frac{v^{2}-2 \omega+1}{2 v(1-\omega)} .
$$

Equations (15) and (16) provide a parameterized curve in the $\left(\gamma_{1}, \gamma_{2}\right)$ plane:

$$
\sin \left(v \gamma_{1}\right)=\frac{v^{2}+2 \omega-1}{2 v \omega} \text { and } \sin \left(v \gamma_{2}\right)=\frac{v^{2}-2 \omega+1}{2 v(1-\omega)}
$$

In order to guarantee feasibility we have to satisfy

$$
-1 \leq \frac{v^{2}+2 \omega-1}{2 v \omega} \leq 1
$$

and

$$
-1 \leq \frac{v^{2}-2 \omega+1}{2 v(1-\omega)} \leq 1
$$

Simple calculation shows that with $\omega>1 / 2$ these relations hold if and only if

$$
2 \omega-1 \leq v \leq 1
$$

From (17) we have four cases for $\gamma_{1}$ and $\gamma_{2}$, since

$$
\gamma_{1}=\frac{1}{v}\left\{\sin ^{-1}\left(\frac{v^{2}+2 \omega-1}{2 v \omega}\right)+2 k \pi\right\}
$$

or

$$
\gamma_{1}=\frac{1}{v}\left\{\pi-\sin ^{-1}\left(\frac{v^{2}+2 \omega-1}{2 v \omega}\right)+2 k \pi\right\}(k=0,1,2, \ldots)
$$

and similarly

$$
\gamma_{2}=\frac{1}{v}\left\{\sin ^{-1}\left(\frac{v^{2}-2 \omega+1}{2 v(1-\omega)}\right)+2 n \pi\right\}
$$

or

$$
\gamma_{2}=\frac{1}{v}\left\{\pi-\sin ^{-1}\left(\frac{v^{2}-2 \omega+1}{2 v(1-\omega)}\right)+2 n \pi\right\}(n=0,1,2, \ldots) .
$$

However from (11) we can see that $\cos \left(v \gamma_{1}\right)$ and $\cos \left(v \gamma_{2}\right)$ must have different signs, so we have only two possibilities:

$$
L_{1}(k, n):\left\{\begin{array}{l}
\gamma_{1}=\frac{1}{v}\left\{\sin ^{-1}\left(\frac{v^{2}+2 \omega-1}{2 v \omega}\right)+2 k \pi\right\} \\
\gamma_{2}=\frac{1}{v}\left\{\pi-\sin ^{-1}\left(\frac{v^{2}-2 \omega+1}{2 v(1-\omega)}\right)+2 n \pi\right\}
\end{array}\right.
$$

and

$$
L_{2}(k, n):\left\{\begin{array}{l}
\gamma_{1}=\frac{1}{v}\left\{\pi-\sin ^{-1}\left(\frac{v^{2}+2 \omega-1}{2 v \omega}\right)+2 k \pi\right\} \\
\gamma_{2}=\frac{1}{v}\left\{\sin ^{-1}\left(\frac{v^{2}-2 \omega+1}{2 v(1-\omega)}\right)+2 n \pi\right\} .
\end{array}\right.
$$

For each $v \in[2 \omega-1,1]$ these equations determine the values of $\gamma_{1}$ and $\gamma_{2}$. At the initial point $v=2 \omega-1$, we have

$$
\frac{v^{2}+2 \omega-1}{2 v \omega}=1 \text { and } \frac{v^{2}-2 \omega+1}{2 v(1-\omega)}=-1
$$

and if $v=1$, then

$$
\frac{v^{2}+2 \omega-1}{2 v \omega}=1 \text { and } \frac{v^{2}-2 \omega+1}{2 v(1-\omega)}=1 .
$$

Therefore the starting point and end point of $L_{1}(k, n)$ are given as

$$
\gamma_{1}^{s}=\frac{1}{2 \omega-1}\left(\frac{\pi}{2}+2 k \pi\right), \gamma_{2}^{s}=\frac{1}{2 \omega-1}\left(\frac{3 \pi}{2}+2 n \pi\right)
$$

and

$$
\gamma_{1}^{e}=\frac{\pi}{2}+2 k \pi \text { and } \gamma_{2}^{e}=\frac{\pi}{2}+2 n \pi
$$

Similarly, the starting and end points of $L_{2}(k, n)$ are as follows:

$$
\gamma_{1}^{S}=\frac{1}{2 \omega-1}\left(\frac{\pi}{2}+2 k \pi\right), \gamma_{2}^{S}=\frac{1}{2 \omega-1}\left(-\frac{\pi}{2}+2 n \pi\right)
$$

and

$$
\gamma_{1}^{E}=\frac{\pi}{2}+2 k \pi \text { and } \gamma_{2}^{E}=\frac{\pi}{2}+2 n \pi
$$

With fixed value of $k, L_{1}(k, n)$ and $L_{2}(k, n)$ have the same end point, however the starting point of $L_{1}(k, n)$ is the same as that of $L_{2}(k, n+1)$. Therefore the segments $L_{1}(k, n)$ and $L_{2}(k, n)$ with fixed $k$ form a continuous curve with $n=0,1,2, \ldots$. They are shown in Figure 1 for 
$k=0$. The curves $L_{1}(0, n)$ are shown in red color and curves $L_{2}(0, n)$ are given in blue.

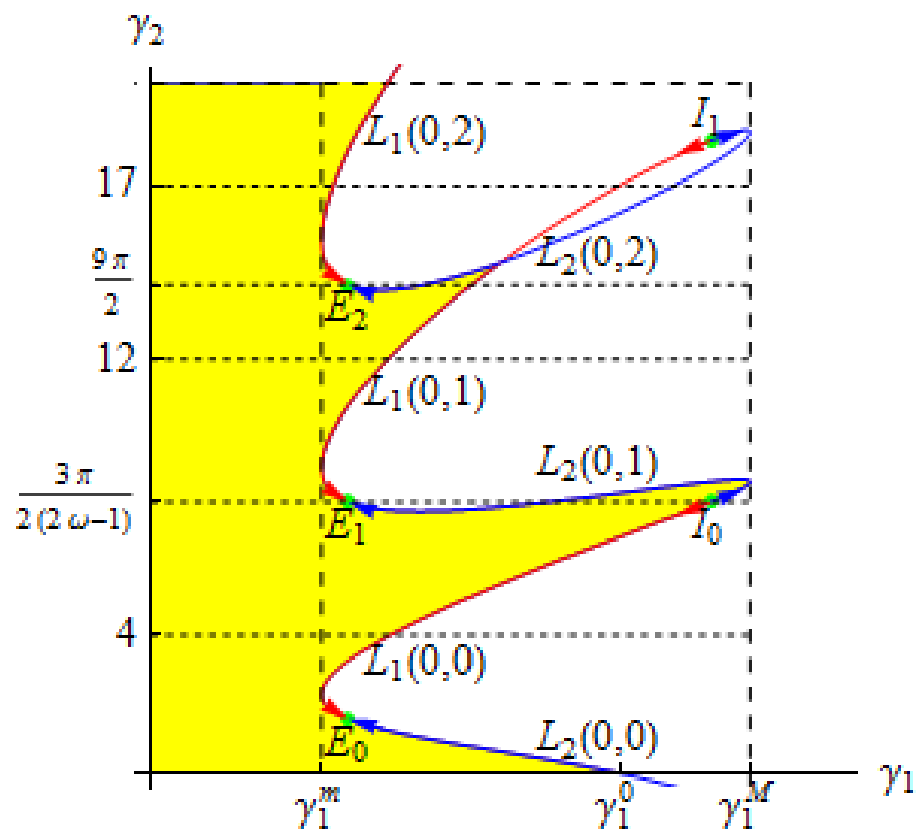

Figure 1. Partition curve in the $\left(\gamma_{1}, \gamma_{2}\right)$ plane with fixing $k=0$

Consider first the segment $L_{1}(k, n)$. Since $\left(v^{2}-2 \omega+1\right) /(2 v(1-\omega))$ is strictly increasing in $v, \gamma_{2}$ is strictly decreasing in $v$. By differentiation

$$
\left.\frac{\partial \gamma_{1}}{\partial v}\right|_{L_{1}}=-\frac{1}{v^{2}}\left(v \gamma_{1}+\tan \left(v \gamma_{2}\right)\right)
$$

Consider next segment $L_{2}(k, n)$, similarly to (22) we can show that

$$
\left.\frac{\partial \gamma_{1}}{\partial v}\right|_{L_{2}}=-\frac{1}{v^{2}}\left(v \gamma_{1}+\tan \left(v \gamma_{2}\right)\right)
$$

which is the same as in $L_{1}(k, n)$, since from (21), $\cos \left(v \gamma_{1}\right)<0$. Similarly

$$
\left.\frac{\partial \gamma_{2}}{\partial v}\right|_{L_{2}}=-\frac{1}{v^{2}}\left(v \gamma_{2}+\tan \left(v \gamma_{1}\right)\right)
$$

where we used again equation (11).

We will next examine the directions of the stability switches on the different segments of the curves $L_{1}(k, n)$ and $L_{2}(k, n)$. We fix the value of $\gamma_{2}$ and select $\gamma_{1}$ as the bifurcation parameter, so the eigenvalues are functions of $\gamma_{1}: \bar{\lambda}=\lambda\left(\gamma_{1}\right)$. By differentiating the characteristic equation (10) implicitly with respect to $\gamma_{1}$ we have

$$
\frac{d \bar{\lambda}}{d \gamma_{1}}+\omega e^{-\bar{\lambda} \gamma_{1}}\left(-\frac{d \bar{\lambda}}{d \gamma_{1}} \gamma_{1}-\bar{\lambda}\right)+(1-\omega) e^{-\bar{\lambda} \gamma_{2}}\left(-\frac{d \bar{\lambda}}{d \gamma_{1}} \gamma_{2}\right)=0
$$

implying that

$$
\frac{d \bar{\lambda}}{d \gamma_{1}}=\frac{\bar{\lambda} \omega e^{-\bar{\lambda} \gamma_{1}}}{1-\omega \gamma_{1} e^{-\bar{\lambda} \gamma_{1}}-(1-\omega) \gamma_{2} e^{-\bar{\lambda} \gamma_{2}}}
$$

From the characteristic equation we have

$$
(1-\omega) e^{-\bar{\lambda} \gamma_{2}}=-\bar{\lambda}-\omega e^{-\bar{\lambda} \gamma_{1}},
$$

so

$$
\frac{d \bar{\lambda}}{d \gamma_{1}}=\frac{\bar{\lambda} \omega e^{-\bar{\lambda} \gamma_{1}}}{1+\bar{\lambda} \gamma_{2}+\omega\left(\gamma_{2}-\gamma_{1}\right) e^{-\bar{\lambda} \gamma_{1}}}
$$

If $\bar{\lambda}=i v$, then

$$
\frac{d \bar{\lambda}}{d \gamma_{1}}=\frac{i v \omega\left(\cos \left(v \gamma_{1}\right)-i \sin \left(v \gamma_{1}\right)\right)}{1+i v \gamma_{2}+\omega\left(\gamma_{2}-\gamma_{1}\right)\left(\cos \left(v \gamma_{1}\right)-i \sin \left(v \gamma_{1}\right)\right)}
$$

and the real part of this expression has the same sign as

$$
\begin{aligned}
& v \omega \sin \left(v \gamma_{1}\right)\left[1+\omega\left(\gamma_{2}-\gamma_{1}\right) \cos \left(v \gamma_{1}\right)\right]+ \\
& v \omega \cos \left(v \gamma_{1}\right)\left[v \gamma_{2}-\omega\left(\gamma_{2}-\gamma_{1}\right) \sin \left(v \gamma_{1}\right)\right] \\
& =v \omega\left[\sin \left(v \gamma_{1}\right)+v \gamma_{2} \cos \left(v \gamma_{1}\right)\right] .
\end{aligned}
$$

Hence 


$$
\operatorname{Re}\left(\frac{d \bar{\lambda}}{d \gamma_{1}}\right) \leq 0 \text { if and only if } \sin \left(v \gamma_{1}\right)+v \gamma_{2} \cos \left(v \gamma_{1}\right) \leq 0
$$

Consider first the case of crossing any segment $L_{1}(k, n)$ from the left. Here $v \gamma_{1} \in(0, \pi / 2]$, so both $\sin \left(v \gamma_{1}\right)$ and $\cos \left(v \gamma_{1}\right)$ are positive. Hence stability is lost everywhere on any segment of $L_{1}(k, n)$. Consider the case when crossing the segments of $L_{2}(k, n)$ from the left. Stability is lost when $\gamma_{2}$ increases in $v$ and stability is gained when $\gamma_{2}$ decreases in $v$. At all intersections with $L_{1}(k, n)$ and $L_{2}(k, n)$ Hopf bifurcation occurs giving the possibility of the birth of limit cycles.

We can also show that at any intersection with $L_{1}(k, n)$ or $L_{2}(k, n)$ the pure complex root is single. Otherwise $\lambda=i v$ would satisfy both equations

$$
\lambda+\omega e^{-\lambda \gamma_{1}}+(1-\omega) e^{-\lambda \gamma_{2}}=0
$$

and

$$
1-\omega \gamma_{1} e^{-\lambda \gamma_{1}}-(1-\omega) \gamma_{2} e^{-\lambda \gamma_{2}}=0,
$$

from which we have

$$
e^{-\lambda \gamma_{1}}=\frac{1+\lambda \gamma_{2}}{\left(\gamma_{1}-\gamma_{2}\right) \omega} \text { and } e^{-\lambda \gamma_{2}}=\frac{-1-\lambda \gamma_{1}}{\left(\gamma_{1}-\gamma_{2}\right)(1-\omega)} .
$$

By substituting $\lambda=i v$ and comparing the real and imaginary parts yield

$$
\sin \left(v \gamma_{1}\right)+v \gamma_{2} \cos \left(v \gamma_{1}\right)=\sin \left(v \gamma_{2}\right)+v \gamma_{1} \cos \left(v \gamma_{2}\right)=0 .
$$

Therefore this intersection is at an extremum in $v$ of a segment $L_{1}(k, n)$ and also at an extremum of a segment $L_{2}(\bar{k}, \bar{n})$ which is impossible.

Assume next that $\omega=1 / 2$. Then equations (11) and (12) imply that

$$
\begin{gathered}
\cos \left(v \gamma_{1}\right)+\cos \left(v \gamma_{2}\right)=0 \\
v-\frac{1}{2}\left(\sin \left(v \gamma_{1}\right)+\sin \left(v \gamma_{2}\right)\right)=0
\end{gathered}
$$

and the curves $L_{1}(k, n)$ and $L_{2}(k, n)$ are simplified as follows:

$$
L_{1}(k, n):\left\{\begin{array}{l}
\gamma_{1}=\frac{1}{v}\left(\sin ^{-1}(v)+2 k \pi\right) \\
\gamma_{2}=\frac{1}{v}\left(\pi-\sin ^{-1}(v)+2 n \pi\right)
\end{array}\right.
$$

and

$$
L_{2}(k, n):\left\{\begin{array}{l}
\gamma_{1}=\frac{1}{v}\left(\pi-\sin ^{-1}(v)+2 k \pi\right) \\
\gamma_{2}=\frac{1}{v}\left(\sin ^{-1}(v)+2 n \pi\right) .
\end{array}\right.
$$

The stability switching curves are shown in Figure 2 in which the stability region is the gray area.

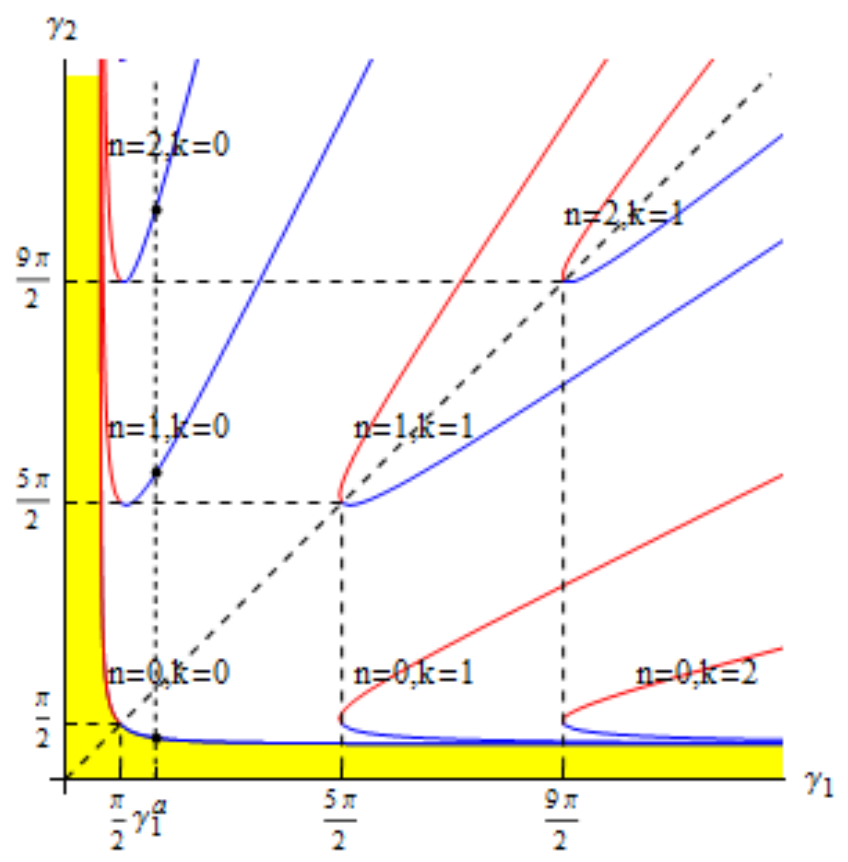

Figure 2. Partition curve in the $\left(\gamma_{1}, \gamma_{2}\right)$ plane with $\omega=1 / 2$ 


\section{Model 2}

In this section we consider a simple dynamic system in macroeconomics, following Matsumoto and Szidarovszky (2015). Based on Philips (1954), we construct the following dynamics model,

$$
\begin{gathered}
C(t)=\alpha Y(t-\eta) \\
I(t)=\varphi(\dot{Y}(\mathrm{t}-\delta)) \\
Y(t)=\int_{0}^{t} \frac{1}{\varepsilon} e^{-\frac{t-\tau}{\varepsilon}} E(\tau) d \tau .
\end{gathered}
$$

Here $C, I$ and $Y$ denote consumption, investment and national income, $\eta>0$ and $\delta>0$ are the consumption delay and investment delay. The first equation is the consumption function, the second equation is the induced investment where the acceleration principle is assumed with $\varphi^{\prime}(Y)>0$ and $\varphi^{\prime \prime}(Y) \neq 0 . E(\tau)=C(\tau)+I(\tau)$ is the total expenditure and the last equation indicates that national income lags behind the expenditure and this delay is of exponential form. Differentiating the last equation with respect to $t$ and substituting delayed consumption and investment into the resultant expression presents a differential equation with two fixed delays,

$$
\varepsilon \frac{d Y(t)}{d t}-\phi\left(\frac{d Y(t-\delta)}{d t}\right)+Y(t)-\alpha Y(t-\eta)=0 .
$$

This is the dynamic model we will analyze. Notice that $Y^{e}=0$ with $d Y^{e}(t) / d t=0$ is the unique stationary equilibrium. To consider its local stability, equation (27) is linearly approximated,

$$
\varepsilon \frac{d Y(t)}{d t}+Y(t)-v \frac{d Y(t-\delta)}{d t}-\alpha Y(t-\eta)=0,
$$

where $v=\varphi^{\prime}(0)$. With the notation

$$
a=\frac{1}{\varepsilon}, b=\frac{v}{\varepsilon}, c=\frac{\alpha}{\varepsilon} \text { and } x=Y
$$

it becomes

$$
\dot{x}(t)+a x(t)-b \dot{x}(t-\delta)-c x(t-\eta)=0 .
$$

Here $a, b, c>0$ and $b<1, c<a$. Similarly to the previous model it is easy to prove that the system is stable without delays and also with a single delay, when either $\delta=0, \eta=0$ or $\delta=\eta$. The corresponding characteristic equation is obtained by substituting an exponential solution, $x(t)=e^{\lambda t} u$,

$$
\lambda+a-b \lambda e^{-\delta \lambda}-c e^{-\eta \lambda}=0 .
$$

Dividing its both sides by $a+\lambda$ and introducing the new functions,

$$
a_{1}(\lambda)=-\frac{b \lambda}{a+\lambda} \text { and } a_{2}(\lambda)=-\frac{c}{a+\lambda}
$$

simplify equation (29),

$$
a(\lambda)=1+a_{1}(\lambda) e^{-\delta \lambda}+a_{2}(\lambda) e^{-\eta \lambda}=0 .
$$

The terms of this function are shown in Figure 3.

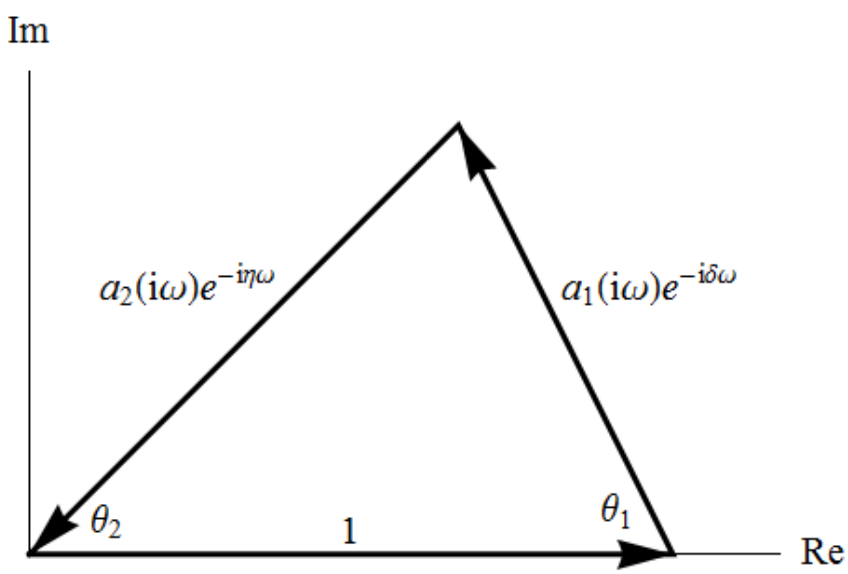

Figure 3. Triangle formed by $1,\left|a_{1}(i \omega)\right|$ and $\left|a_{2}(i \omega)\right|$

Suppose that $\lambda=i \omega$ with $\omega>0$, then

$$
a_{1}(i \omega)=-\frac{b \omega^{2}}{a^{2}+\omega^{2}}-i \frac{a b \omega}{a^{2}+\omega^{2}}
$$

and

$$
a_{2}(i \omega)=-\frac{a c}{a^{2}+\omega^{2}}+i \frac{c \omega}{a^{2}+\omega^{2}}
$$

Their absolute values are

$$
\left|a_{1}(i \omega)\right|=\frac{b \omega}{\sqrt{a^{2}+\omega^{2}}} \text { and }\left|a_{2}(i \omega)\right|=\frac{c}{\sqrt{a^{2}+\omega^{2}}}
$$

and their arguments are

$$
\arg \left(a_{1}(i \omega)\right)=\tan ^{-1}\left(\frac{a}{\omega}\right)+\pi \text { and } \arg \left(a_{2}(i \omega)\right)=\pi-\tan ^{-1}\left(\frac{\omega}{a}\right)
$$

The triangle can be above the real line and also under the real line. In the two cases the following relations hold for angles $\theta_{1}$ and $\theta_{2}$ :

$$
\arg \left(a_{1}(i \omega)\right)-\delta \omega \pm \theta_{1}=\pi+2 n \pi
$$

and

$$
\arg \left(a_{2}(i \omega)\right)-\eta \omega \mp \theta_{2}=\pi+2 m \pi \text {. }
$$

In a triangle consisting of three line segments, the length of the sum of any two adjacent line segments is not shorter than the length of the remaining line segment, 


$$
\begin{aligned}
& 1 \leq\left|a_{1}(i \omega)\right|+\left|a_{2}(i \omega)\right|, \\
& \left|a_{1}(i \omega)\right| \leq 1+\left|a_{2}(i \omega)\right|
\end{aligned}
$$

and

$$
\left|a_{2}(i \omega)\right| \leq 1+\left|a_{1}(i \omega)\right|
$$

Substituting the absolute values renders these three conditions to the following two conditions,

$$
f(\omega)=\left(1-b^{2}\right) \omega^{2}-2 b c \omega+a^{2}-c^{2} \leq 0
$$

and

$$
g(\omega)=\left(1-b^{2}\right) \omega^{2}+2 b c \omega+a^{2}-c^{2} \geq 0 .
$$

Both $f(\omega)$ and $g(\omega)$ have the same discriminant,

$$
D=4\left[c^{2}-a^{2}\left(1-b^{2}\right)\right] .
$$

In the following we draw attention to the case of $D>0$, otherwise $f(\omega)>0$ for all $\omega$ implying no stability switch. Solving $g(\omega)=0$ gives the solutions

$\omega_{1}=\frac{-b c-\sqrt{c^{2}-a^{2}\left(1-b^{2}\right)}}{1-b^{2}}$ and $\omega_{2}=\frac{-b c+\sqrt{c^{2}-a^{2}\left(1-b^{2}\right)}}{1-b^{2}}$

and so does solving $f(\omega)=0$,

$$
\omega_{3}=\frac{b c-\sqrt{c^{2}-a^{2}\left(1-b^{2}\right)}}{1-b^{2}} \text { and } \omega_{4}=\frac{b c+\sqrt{c^{2}-a^{2}\left(1-b^{2}\right)}}{1-b^{2}} \text {. }
$$

Since both $\omega_{1}$ and $\omega_{2}$ are negative and both $\omega_{3}$ and $\omega_{4}$ are positive, the two conditions, $f(\omega) \leq 0$ and $g(\omega) \geq 0$, are satisfied when $\omega$ is in interval $\left[\omega_{3}, \omega_{4}\right]$.

The internal angles, $\theta_{1}$ and $\theta_{2}$, of the triangle in Figure 3 can be calculated by the law of cosine as

$$
\theta_{1}(\omega)=\cos ^{-1}\left(\frac{a^{2}+\left(1+b^{2}\right) \omega^{2}-c^{2}}{2 b \omega \sqrt{a^{2}+\omega^{2}}}\right)
$$

and

$$
\theta_{2}(\omega)=\cos ^{-1}\left(\frac{a^{2}+\left(1-b^{2}\right) \omega^{2}+c^{2}}{2 c \sqrt{a^{2}+\omega^{2}}}\right)
$$

Solving equations (33) and (34) for $\delta$ and $\eta$ yields

$$
\delta^{ \pm}(\omega, k)=\frac{1}{\omega}\left[\tan ^{-1}\left(\frac{a}{\omega}\right)+\pi+(2 k-1) \pi \pm \theta_{1}(\omega)\right]
$$

and

$$
\eta^{\mp}(\omega, k)=\frac{1}{\omega}\left[-\tan ^{-1}\left(\frac{\omega}{a}\right)+\pi+(2 n-1) \pi \mp \theta_{2}(\omega)\right],
$$

so we have again two stability switching curves with fixed values of $k$ and $n$,

$$
L_{1}(k, n)=\left\{\delta^{+}(\omega, k), \eta^{-}(\omega, n)\right\}
$$

and

$$
L_{2}(k, n)=\left\{\delta^{-}(\omega, k), \eta^{+}(\omega, n)\right\}
$$

They are shown in Figure 4 for the case of $k=n=1$. They have the same initial point $S$ and arrive at the same end point $E$ as $\omega$ increases from $\omega_{3}$ to $\omega_{4}$. With fixed $\eta=\eta^{0}$ by increasing the value of $\delta$, stability is lost at point $A$ and regained at point $B$. These curves are shifted to the right by increasing the value of $k$ and up by increasing the value of $n$.

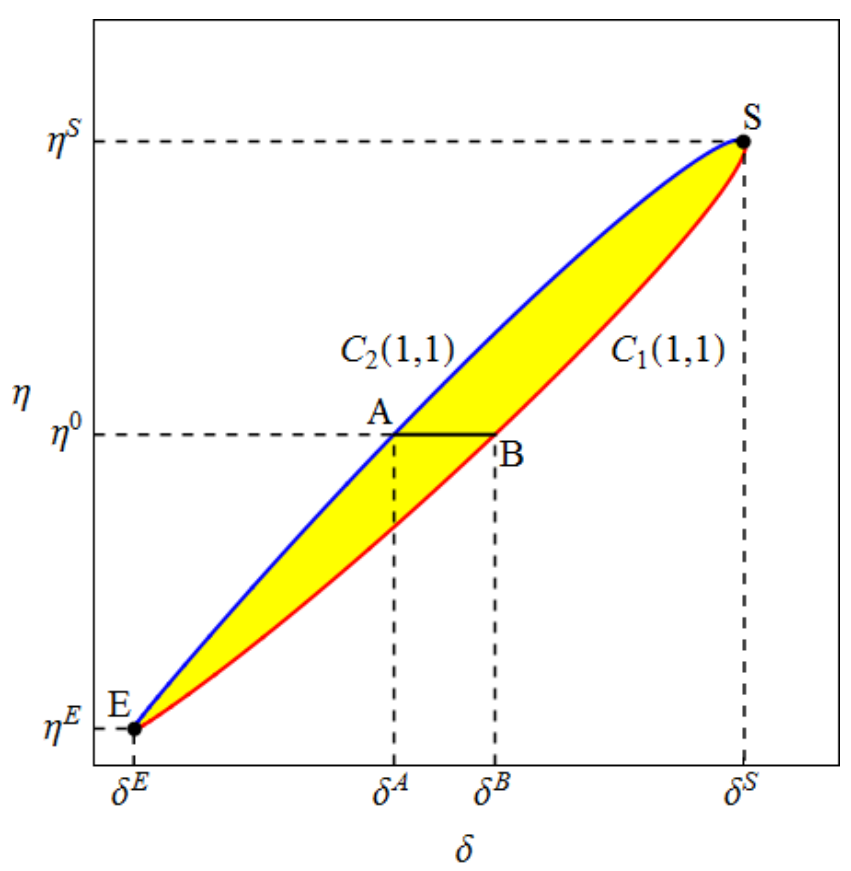

Figure 4. Partition curve with $k=1$ and $n=1$

\section{Simulations}

In the first case, Figure 5(A) shows the six cigar-shaped domains obtained for $k=0,1,2,3,4,5$ and $n=1$ and their lower parts are colored in yellow. We fix $\eta=1$ and increase $\delta$ from 1 to 4 along the dotted horizontal line. The system is stable until $\delta=\delta_{1}$, when stability is lost. It is regained at $\delta=\delta_{2}$ and system remains stable until $\delta=\delta_{3}$ where stability is lost, and regained again at $\delta=\delta_{4}$, and so on. So stability is lost at points $\delta_{1}, \delta_{3}, \delta_{5}$ and $\delta_{7}$ and stability is regained at points $\delta_{2}, \delta_{4}$ and $\delta_{6}$. The bifurcation diagram shown in Figure $5(\mathrm{~B})$ well demonstrates 
these observations.

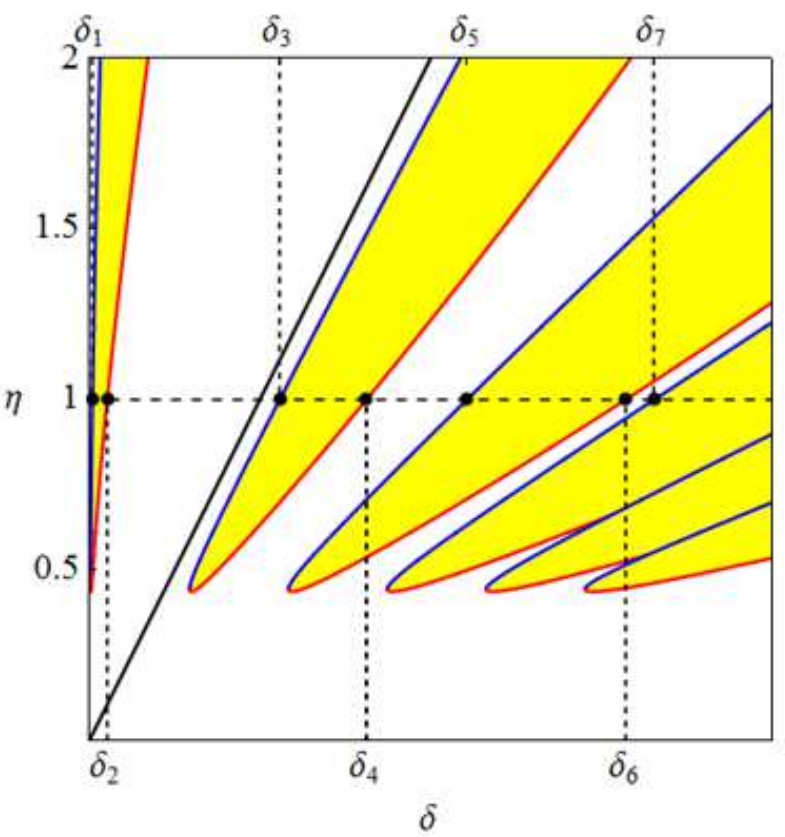

(A) Partition curve

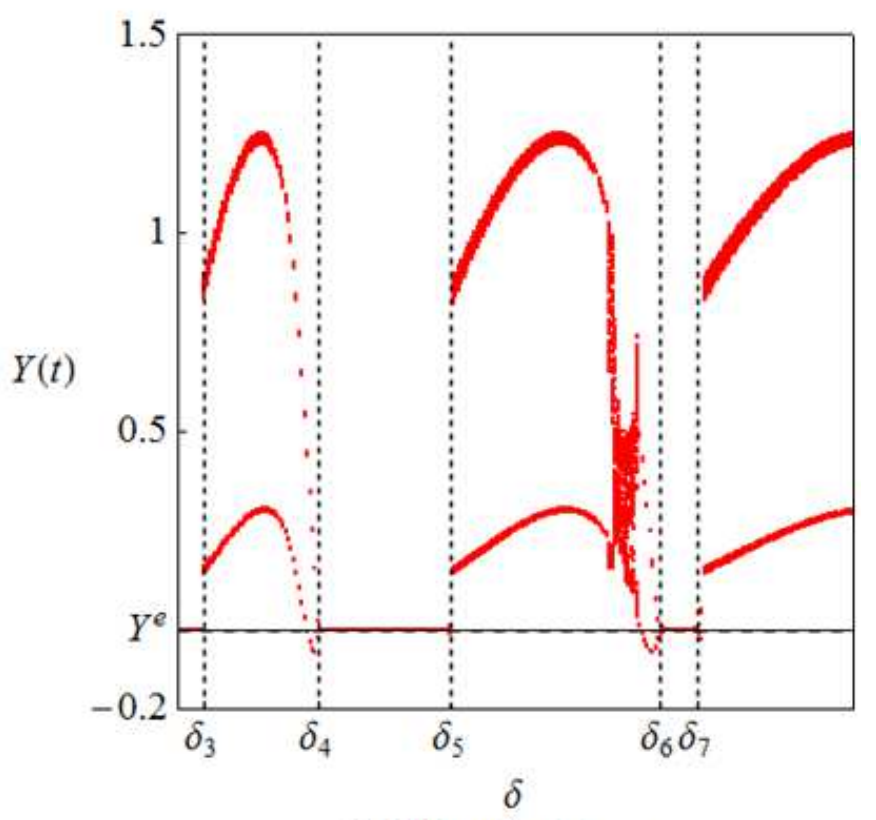

(B) Bifurcation diagram

Figure 5. Stability switches with $\eta=1$

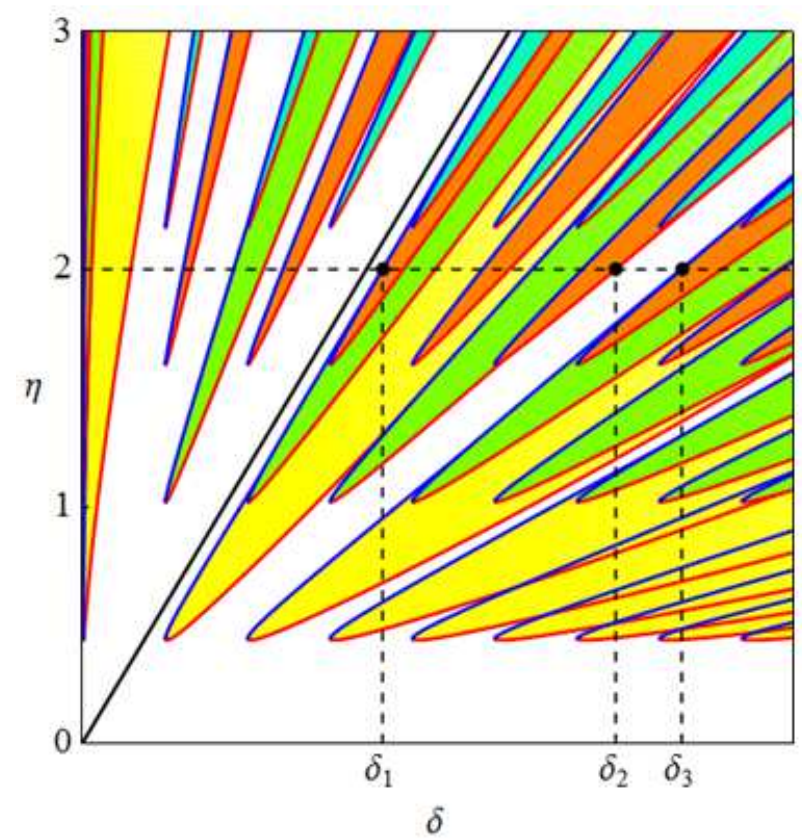

(A)Partition curve

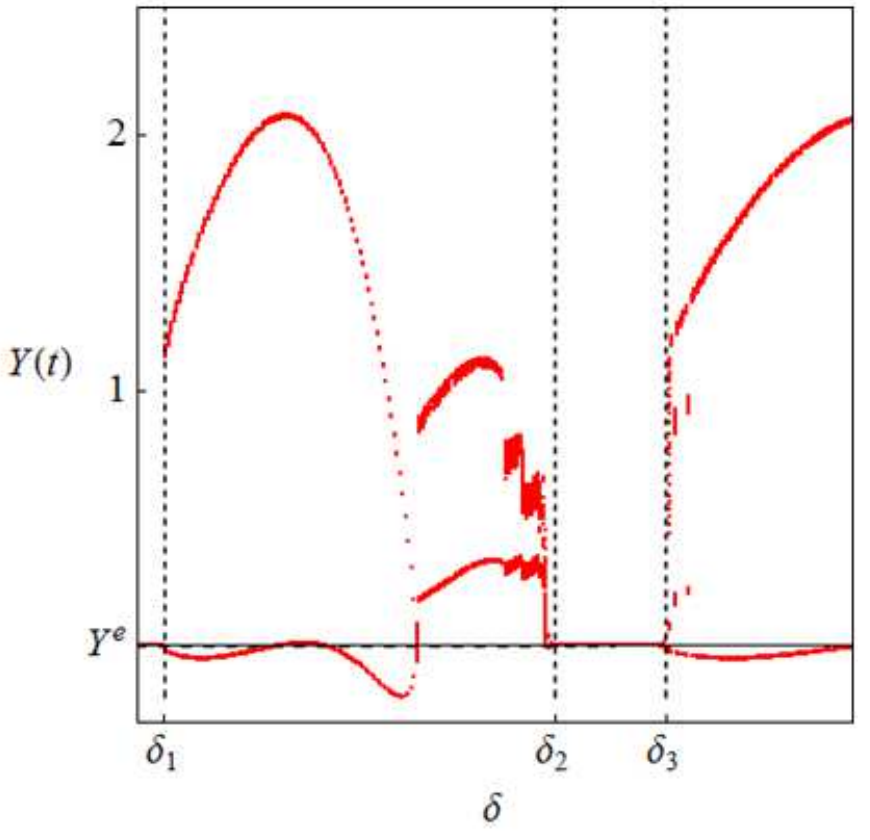

(B) Bifurcation diagram

Figure 6. Stability switches with $\eta=2$

In the second simulation we illustrate the curves $L_{1}(k, n)$ and $L_{2}(k, n)$ for $k=0,1, \ldots, 8$ and $n=1,2,3,4$ in Figure 6(A). The yellow domains are surrounded by $L_{1}(k, 1)$ and $L_{2}(k, 1)$, which are the same as in Figure 5(A). The green regions are surrounded by $L_{1}(k, 2)$ and $L_{2}(k, 2)$, and the orange and blue regions by $L_{1}(k, 3)$ and $L_{2}(k, 3)$ and by $L_{1}(k, 4)$ and $L_{2}(k, 4)$, respectively. The value of $\eta=2$ is now selected. The dotted horizontal line crosses the stability switching curves many times, but not all intersections are stability switches. For example, between $\delta_{1}$ and $\delta_{2}$ the system is unstable regardless of several intersections between them. At $\delta=\delta_{2}$ stability is regained, and lost again at $\delta=\delta_{3}$. The bifurcation diagram shown in Figure 6(B) well illustrates these findings.

Let $(\delta, \eta)$ be any point in the positive quadrant and not on 
the stability switching curves and consider the line segment connecting points $(0, \eta)$ and $(\delta, \eta)$. Let $L$ be the number of intersections of this segment with the stability switching curves with stability loss and $G$ the number of intersections with stability gain. The system is stable for $(\delta, \eta)$ if $G \geq L$, otherwise unstable.

\section{Conclusions}

Two particular economic models were examined. Both are first order ordinary differential equations with two delays. The stability switching curves were first determined where an eigenvalue is pure complex, and then the stability and instability regions were demonstrated. In the first case an elementary analytic approach was used, and in the second case a geometric approach was shown. This approach could be also used for solving the first model as well, however the more simple analytic approach cannot be used for the second model without major changes.

\section{Acknowledgements}

The first author highly appreciates the financial supports from the MEXT-Supported Program for the Strategic Research Foundation at Private Universities 2013-207, the Japan Society for the Promotion of Science (Grant-in-Aid for Scientific Research (C) 24530202, 25380238 and 26380316). The second author appreciates the hospitality of the Department of Economics of Chuo University, Tokyo while the research leading to this paper was conducted. The usual disclaimers apply. This paper is dedicated to Professor Toshikazu Ito on the occasion of his retirement at Ryukoku University.

\section{References}

[1] Bellman, R. and Cooke, K. (1963), Differential-Difference Equations. Academic Press, New York.

[2] Gu, K., Niculescu, S. and Chen, J. (2005), On Stability Crossing Curves for General Systems with Two Delays. Journal of Mathematical Analysis and Applications, 311, 231-252.

[3] Hayes, N. D. (1950), Roots of the Transcendental Equation Associated with a Certain Difference-Differential Equation. Journal of the London Mathematical Society, 25, 226-232.

[4] Hale, J. (1979), Nonlinear Oscillations in Equations with Delays. In Nonlinear Oscillations in Biology (F. C. Hoppenstadt, ed.). Lectures in Applied Mathematics, 17, American Mathematical Society, 157-185.

[5] Hale, J. and Huang, W. (1993), Global Geometry of the Stable Regions for Two Delay Differential Equations. Journal of Mathematical Analysis and Applications, 178, 344-362.

[6] Matsumoto, A. and Szidarovszky, F. (2015), Nonlinear Multiplier-Acceelerator Model with Investment and Consumption Delay. Structural Change and Economic Dynamics, 33, 1-9.

[7] Matsumoto, A. and Szidarovszky, F. (2013a), An Elementary Study of a Class of Dynamic Systems with Single Delay. CUBO A Mathematical Journal, 15, 1-7.

[8] Matsumoto, A. and Szidarovszky, F. (2013b), Learning in Monopolies with Delayed Price Information. IERCH DP. No.203, Institute of Economic Research, Chuo University.

[9] Matsumoto, A. and Szidarovszky, F. (2012), An Elementary Study of a Class of Dynamic Systems with Two Time Delays. CUBO A Mathematical Journal, 14, 103-113.

[10] Phillips, A. (1954), Stabilization Policy in a Closed Economy. Economic Journal, 64, 290-323. 